\title{
BNP screening and collaborative care can help to prevent heart failure
}

Control of cardiovascular risk factors is far from ideal throughout the world, and the prevalence of heart failure is expected to increase substantially over the next 20 years. The STOP-HF investigators, therefore, tested a new strategy for prevention of heart failure in a prospective, randomized trial involving a broad community cohort.

Patients, recruited from 39 primary care facilities in Ireland, were aged $>40$ years and had a history of one or more cardiovascular risk factors. Usual primary care, which involved advice on lifestyle modification and risk-factor intervention, was provided to 677 patients. Another 697 patients underwent BNP screening and, if their BNP concentration was $\geq 50 \mathrm{pg} / \mathrm{ml}$, were referred to a specialist cardiovascular service for further investigation and management. Mean follow-up was 4.2 years.

The primary end point-left ventricular dysfunction, newly diagnosed heart failure, or both-occurred in $8.7 \%$ of the control group and $5.3 \%$ of patients in the group that received $\mathrm{BNP}$ screening and collaborative care (OR 0.55, 95\% CI 0.37-0.82, $P=0.003$ ).

Fewer of the screening group were admitted to hospital for major adverse cardiovascular events $(7.3 \%$ vs $10.5 \%$ in controls; OR 0.69 , $95 \%$ CI $0.49-0.98, P=0.04)$. Studies to assess the cost-effectiveness of the strategy, as well as effects on quality of life, are ongoing.

In an editorial that accompanied the trial report, Adrian Hernandez concludes that "both screening and aggressive risk factor modification appear to be necessary tools in the quest to prevent heart failure". He suggests that future trials aimed at preventing heart failure should be focused on outcomes to which patients can easily relate (for example, exercise capacity, symptoms, health status, and survival).

Bryony M. Mearns

Original article Ledwidge, M. et al. Natriuretic peptidebased screening and collaborative care for heart failure: the STOP-HF randomized trial. JAMA 310, 66-74 (2013) 